\title{
c-Jun N-terminal kinase inhibition attenuates early brain injury induced neuronal apoptosis via decreasing p53 phosphorylation and mitochondrial apoptotic pathway activation in subarachnoid hemorrhage rats
}

\author{
GENG-QIANG LING ${ }^{1,2}$, XIAN-FENG LI ${ }^{1}$, XU-HUI LEI ${ }^{1}$, \\ ZHEN-YU WANG ${ }^{1}$, DONG-YING MA ${ }^{1}$, YUE-NA WANG ${ }^{1}$ and WEI YE ${ }^{1,3}$ \\ ${ }^{1}$ Department of Neurosurgery, The Second Affiliated Hospital of Harbin Medical University, Harbin, Heilongiang 150086;
${ }^{2}$ Department of Neurosurgery, The Seventh Affiliated Hospital of Sun Yat-sen University, Shenzhen, Guangdong 518107;
${ }^{3}$ Neuroscience Institute, Heilongjiang Academy of Medical Sciences, Harbin, Heilongjiang 150086, P.R. China
}

Received March 8, 2018; Accepted October 1, 2018

DOI: $10.3892 / \mathrm{mmr} .2018 .9640$

\begin{abstract}
Early brain injury (EBI)-induced neuronal apoptosis is primarily responsible for the subsequent complications of aneurysmal subarachnoid hemorrhage (aSAH), which may increase the risk of mortality in patients with aSAH. c-Jun $\mathrm{N}$-terminal kinase (JNK) has been demonstrated to be a promoter of EBI-induced cell apoptosis, although the mechanism has yet to be fully elucidated. The present study aimed to explore whether the role of JNK1 is associated with tumor protein $\mathrm{p} 53$ (p53), which is one of the most important factor that triggers cell apoptosis. JNK1 expression was downregulated via in vivo small interfering RNA transfection in an aSAH rat model in order to assess differences in the behavior, survival times, morphology and genetics of the experimental animals. The results revealed that JNK1 inhibition improved the neurological scores and survival times of SAH rats by interrupting cascaded neuronal apoptosis. The interruption of EBI-induced neuronal apoptosis may originate from a decrease in the level of p53 phosphorylation and deactivation of the downstream mitochondrial apoptotic pathway. Taken together, these results suggest that JNK1 may be a promising target for improving the prognosis of patients with aSAH.
\end{abstract}

\section{Introduction}

Aneurysmal subarachnoid hemorrhage (aSAH) is a serious disease that subsequently leads to a series of severe

Correspondence to: Professor Wei Ye, Department of Neurosurgery, The Second Affiliated Hospital of Harbin Medical University, 246 Xuefu Road, Harbin, Heilongjiang 150086, P.R. China

E-mail: yeweizhuren@163.com

Key words: aneurysmal subarachnoid hemorrhage, early brain injury, apoptosis, c-Jun $\mathrm{N}$-terminal kinase, tumor protein 53 complications, including cephaledema, vasospasm, hydrocephalus and seizure (1). Previously, it was suggested that delayed cerebral ischemia due to vasospasm was the predominant cause of subacute complications of aSAH (2). However, an antagonist of vasospasm failed to decrease patient mortality rates or to improve the neurological outcomes (3). Previously, early brain injury (EBI) has been increasingly considered to be responsible for the unfavorable outcome of aSAH (4). EBI is defined according to the pathophysiological status of a patient typically occurring within $72 \mathrm{~h}$ following an aneurysmal rupture, along with subsequent complications, including brain edema, vasospastic ischemia and delayed ischemic neurological deficits (5). In this phenomenon, neuronal apoptosis has been suggested to be the key process leading to numerous pathological events (6). Therefore, inhibition of neuronal apoptosis in the early stages of aSAH may be beneficial for the treatment of EBI.

Elucidation of the mechanism of aSAH-induced apoptosis has been an area of investigation for several years. Unlike cell apoptosis in a model of focal ischemia, aSAH-induced apoptosis was partly attributed to the complex interaction of injurious blood-derived factors (7). Essentially, this process comprises 4 primary signaling pathways: The death receptor/tumor protein p53 (p53) pathway; the caspase-dependent and -independent pathways; and the mitochondrial pathway (5). The death receptor/p53 signaling pathway, also referred to as the extrinsic apoptotic pathway, is considered to be particularly important in cerebral vasospasm following aSAH (8). The caspase-dependent cascade is primarily associated with ischemia, whereas the caspase-independent cascade is more closely associated with neurotoxin-induced apoptosis (9). The mitochondrial pathway, also termed the intrinsic pathway, is likely to function as a signaling pathway downstream of p53 activation, which is elicited by DNA damage (10). p53 appears to serve a central role as a cross-signaling molecule in aSAH-induced apoptosis.

It has been well established that the c-Jun N-terminal kinase (JNK)-associated signaling pathway serves a 
particularly important role in cell apoptosis. The JNKs, as members of the mitogen-activated protein kinase family, are encoded by three genes (JNK1, JNK2 and JNK3) and may be activated by cellular environmental stresses, inflammatory cytokines and growth factors (11). Downstream factors of the JNK signaling pathway comprise c-Jun, activating transcription factor 2 (ATF-2), p53, ETS domain-containing protein Elk1, mothers against decapentaplegic homolog 4, signal transducer and activator of transcription 3 and nuclear factor of activated T cells 4 (12). In the case of apoptosis induced by artificial DNA damage, JNK was demonstrated to be able to activate p53 by phosphorylation in vivo (13). Furthermore, a subsequent study on chemically-induced apoptosis in rat livers suggested that the JNK1 gene was associated with p53 activation (14). In the case of EBI, Dai et al (15) demonstrated that sp600125, a JNK-specific inhibitor, was able to ameliorate EBI by decreasing levels of neuronal apoptosis in the brain tissue of rats. However, the association between JNK1 and p53 in the EBI process remains unclear. Therefore, a preliminary investigation of RNA interference towards the JNK1 gene was performed in the present study to investigate how JNK1 may interact with p53 in neuronal apoptosis induced by EBI.

\section{Materials and methods}

Animal preparation and grouping. A total of 50 male Sprague-Dawley (SD) rats (Beijing Vital River Laboratory Animal Technology Co., Ltd., Beijing, China) weighing 300-350 g were used in the present study. The rats were fed in a temperature- and humidity-controlled animal center with ad libitum access to water and food. They were divided randomly into three groups, termed the sham group $(n=10)$, scramble group $(n=20)$ and siRNA group $(n=20)$. Subsequently, 10 rats from the scramble and the siRNA groups were monitored for survival analysis. All procedures were approved by Ethics Committee of the Second Affiliated Hospital of Harbin Medical University (Harbin, China) and were conducted in accordance with the Guide for the Care and Use of Laboratory Animals by the National Institutes of Health (Bethesda, MD, USA) (16).

In vivo RNA interference (RNAi) prior to reverse transcription quantitative polymerase ( $R T-q P C R)$ verification. The rats were injected with JNK1 small interfering RNA (siRNA) (Shanghai GeneChem Co., Ltd., Shanghai, China) via the caudal vein, together with Entranster-in vivo RNA transfection reagent (cat. no. 18668-11-1) or Entranster-in vivo DNA transfection reagent (cat. no. 18668-11-2; all from Engreen Biosystem, Ltd., Beijing, China) respectively, according to the manufacturer's protocol, at a dosage of $3.0 \mathrm{mg} / \mathrm{kg}$ daily for 3 days. The sequences of the injected siRNAs were as follows: siRNA, sense, 5'-AAGCCCAGTAATATAGTAGTA-3', and antisense, 5'-ACGTGACACGTTCGGAGAATT-3'; scramble siRNA, sense, 5'-AATTCTCCGAACGTGTCACGT-3', and antisense, 5'-ACGTGACACGTTCGGAGAATT-3'. After a $24 \mathrm{~h}$ period, the rats were sacrificed for JNK1 transcript detection in hippocampal neuronal tissue using RT-qPCR.

Establishment of the rat SAH model and preparation of brain samples. The experimental rat SAH model was established by autologous arterial blood injection into the pre-chiasmatic cistern, as described previously (17), but with certain modifications. Briefly, the rats were anesthetized with $10 \%$ chloral hydrate (300 mg/kg body weight) intraperitoneally (IP). They were subsequently fixed in a stereotaxic instrument. A microinjector was placed in the sagittal plane with a tilt angle of $45^{\circ}$. The entry point was $5 \mathrm{~mm}$ posterior to the external occipital protuberance in the midline. A longitudinal incision of $1.5 \mathrm{~cm}$ was made along the midline, with the entry point in the center. The atlantooccipital membrane was then exposed by removing part of the occipital bone surrounding the foramen magnum. The tip of the microinjector was lowered to penetrate the atlantooccipital membrane, and then inserted $2-3 \mathrm{~mm}$ into the pre-chiasmatic cistern. A total of $300 \mu 1$ non-heparinized fresh autologous arterial blood was aseptically injected into the pre-chiasmatic cistern slowly for $3 \mathrm{~min}$. To maintain fluid balance, all rats were injected with $2 \mathrm{ml} 0.9 \% \mathrm{NaCl}$ subcutaneously. The rats were then returned to their cages, and free access to food and water was provided following their recovery from anesthesia. In this model, the inferior basal temporal lobe of the SAH group was stained with blood. The rats in the sham group underwent the identical procedure with the exception of the blood injection, and these rats were sacrificed $24 \mathrm{~h}$ following the operation. All the rats in the present study were euthanized by cervical dislocation following anesthesia with $10 \%$ chloral hydrate (300 mg/kg body weight) IP. Brain samples were collected following intracardial perfusion with $4 \%$ paraformaldehyde at $4^{\circ} \mathrm{C}$. The samples were subsequently immersed into $4 \%$ paraformaldehyde at $4^{\circ} \mathrm{C}$ for at least $48 \mathrm{~h}$ prior to subsequent use in the histological analyses.

Terminal deoxynucleotidyl-transferase-mediated dUTP nick-end labeling (TUNEL) assay. TUNEL assays were performed using a TUNEL detection kit, following the manufacturer's protocol (cat. no. WLA029; Wanleibio Co., Ltd., Shenyang, China). In brief, previously fixed hippocampus tissue was dehydrated in graded ethyl alcohol (70, 80,90 and $100 \%$ ), embedded in paraffin, sliced into $5 \mu \mathrm{m}$ sections, and rehydrated (100, 95, 85 and 75\%). The slides were subsequently incubated with $50 \mu 1$ TUNEL reaction mixture for $1 \mathrm{~h}$ at $37^{\circ} \mathrm{C}$ in the dark. Slides were then developed with 3,3'-diaminobenzidine (DAB) and counterstained with $0.2 \%$ hematoxylin for $3 \mathrm{~min}$ at room temperature, and finally mounted in Neutral balsam mounting medium (cat. no. g8590; Shanghai Haoran Biological Technology Co., Ltd., Shanghai, China). The apoptotic cells were identified as those with hyperchromatic nuclei, and counted under a light microscope by an investigator blinded to the grouping. The extent of brain damage was evaluated by determining the average percentage of apoptotic cells in each section counted at magnification, $\mathrm{x} 400$. A total of three sections from each animal were used for quantification. The final average percentages of apoptotic cells of the sections were recorded for analysis.

$R T-q P C R$. Tissues were stored prior to RT-qPCR analysis at $-80^{\circ} \mathrm{C}$. Total RNA was extracted using TRIzol ${ }^{\circledR}$ reagent (Thermo Fisher Scientific, Inc., Waltham, MA, USA). RT was performed using M-MLV Reverse Transcriptase (cat. no. 28025021; Invitrogen; Thermo Fisher Scientific, Inc.). Briefly, $1 \mu \mathrm{l}$ oligo (dT) $)_{15}$ primer (cat. no. C1101-20; Promega Corporation, Madison, WI, USA), $1 \mu 1$ random primers 
(cat. no. C1181; Promega Corporation), $2 \mu \mathrm{l}$ of $2.5 \mathrm{mM}$ dNTP (cat. no. U1511; Promega Corporation) were mixed with $\mathrm{dd}_{2} \mathrm{O}$ in a final reaction volume of $14.5 \mu \mathrm{l}$, and heated to $70^{\circ} \mathrm{C}$ for $5 \mathrm{~min}$ and then cooled on ice for $2 \mathrm{~min}$. Subsequently, $4 \mu 15 \mathrm{X}$ First Strand Buffer [250 mmol/1 Tris-Cl (pH 8.3), 375 mmol/l $\left.\mathrm{KCl}, 15 \mathrm{mmol} / 1 \mathrm{MgCl}_{2}\right], 0.5 \mu \mathrm{l} \mathrm{RNasin}^{\circledR}$ ribonuclease inhibitors (cat. no. N2111; Promega Corporation) and $1 \mu \mathrm{l}$ M-MLV were added. The temperature protocol was as follows: $25^{\circ} \mathrm{C}$ for $10 \mathrm{~min}, 42^{\circ} \mathrm{C}$ for $50 \mathrm{~min}$ and $95^{\circ} \mathrm{C}$ for $5 \mathrm{~min}$. The transcriptional levels of genes were subsequently assessed using a SYBR Green PCR Master mix kit (Applied Biosystems; Thermo Fisher Scientific, Inc.). The sequences of the primers used are summarized in Table I. $\beta$-actin was used as an internal control. The thermocycling conditions were as follows: $94^{\circ} \mathrm{C}$ for $10 \mathrm{~min}$, then $41 \mathrm{cycles}$ of $94^{\circ} \mathrm{C}$ for $10 \mathrm{sec}, 60^{\circ} \mathrm{C}$ for $20 \mathrm{sec}$ and $72^{\circ} \mathrm{C}$ for $30 \mathrm{sec}$, followed by $72^{\circ} \mathrm{C}$ for $2.5 \mathrm{~min}, 40^{\circ} \mathrm{C}$ for $5.5 \mathrm{~min}$, and a melting temperature gradient from 60 to $94^{\circ} \mathrm{C}$ ( $1 \mathrm{sec}$ for every $1.0^{\circ} \mathrm{C}$ rise; $25^{\circ} \mathrm{C}$ for $1 \mathrm{~min}$ ). RT-qPCR products were assessed using an Exicycler ${ }^{\mathrm{TM}} 96$ fluorescence quantitative instrument (Bioneer Corporation, Daejeon, Korea). Data obtained for each of the experimental groups were recorded as fold change values relative to the control group (the mean $2^{-\Delta \Delta \mathrm{Cq}} \pm$ standard error of the mean) (18).

Western blot analysis. Protein concentrations were determined using a bicinchoninic acid (BCA) protein assay kit (Thermo Fisher Scientific, Inc.). Equal amounts of total cellular protein $(40 \mu \mathrm{g} / \mathrm{lane})$ were resolved by $15 \%$ SDS-PAGE, subsequently electro-transferred onto a polyvinylidene fluoride membrane (cat. no. IPVH00010; EMD Millipore, Billerica, MA, USA), and blocked with 5\% non-fat dry milk in TBS/Tween (TBST) solution $(20 \mathrm{mM}$ Tris- $\mathrm{HCl}, 137 \mathrm{mM} \mathrm{NaCl}$ and $0.1 \%$ Tween-20, $\mathrm{pH}$ 7.4) for $2 \mathrm{~h}$ at room temperature. The membranes were incubated with rabbit monoclonal or polyclonal antibodies (all at 1:1,000 dilution) against phosphorylated (p)-p53 (cat. no. WL01813; Wanleibio, Co., Ltd.), phosphorylated (p)-JNK (cat. no. WL02504; Wanleibio Co., Ltd.), Bcl-2-associated X protein (Bax; cat. no. A00183; Wuhan Boster Biological Technology, Ltd., Wuhan, China), B-cell lymphoma 2 (Bcl-2; cat. no. WL01556; Wanleibio Co., Ltd.), p-mitogen-activated protein kinase 11 (p-p38; cat. no. WLP1576; Wanleibio Co., Ltd.), nuclear factor $\kappa$-light-chain-enhancer of activated B-cells (NF-кB; cat. no. WL01980; Wanleibio Co., Ltd.) or cleaved caspase-3 (cat. no. WL01992; Wanleibio Co., Ltd.) diluted in TBST overnight at $4^{\circ} \mathrm{C}$. The membranes were also probed with $\beta$-actin antibody (cat. no. WL01845; Wanleibio Co., Ltd.) as an internal control. The blots were subsequently incubated with the corresponding horseradish peroxidase-conjugated secondary antibody (goat-anti-rabbit immunoglobulin G antibody; cat. no. WLA023; Wanleibio Co., Ltd.; 1:5,000 dilution) for $1 \mathrm{~h}$ at room temperature. Immunoreactive proteins were detected using enhanced chemiluminescence western blotting substrate (Thermo Fisher Scientific, Inc.). Densitometric analysis was performed using Gel-Pro Analyzer 6.0 (Media Cybernetics, Inc., Rockville, MD, USA).

Histological assay. Previously fixed rat brains were dehydrated using graded ethyl alcohol (70, 80, 90 and 100\%), embedded in paraffin, sliced into $5 \mu \mathrm{m}$ sections, and stained with $0.5 \%$ Cresyl Violet (cat. no. 71044080; Sinopharm Chemical Reagent Co., Ltd.,Beijing, China) at room temperature for $10 \mathrm{~min}$. The assessed region was in the inferior basal temporal lobe (portrayed by the area of black ovals in Fig. 2). Cresyl Violet-stained neuronal cell bodies (Nissl bodies) were counted. A total of 5 random fields at magnification, $\mathrm{x} 400$ in each coronary section were selected, and the mean number of intact neurons in the five fields of view was calculated. A total of 4 sections from each animal were used for quantification. The final average number of the 4 sections was regarded as the data value for each sample.

Neurological scoring. The scores of appetite, activity and neurological deficits of the rats (Table II) were recorded as examinations of their behavioral activity, as described previously (19), which were performed by an investigator blinded to the study groups at 24 and $72 \mathrm{~h}$ following SAH. The scores of 6 rats in each group were calculated.

Survival analysis. Overall survival was recorded as the time from the date of the rats being subjected to blood injection into the pre-chiasmatic cistern to the date of mortality. The observational period was 30 days.

Statistical analysis. Data were recorded as the mean \pm standard deviation. SPSS 17.0 was used to perform statistical analysis (SPSS, Inc., Chicago, IL, USA). One-way analysis of variance (ANOVA), followed by either the least significant difference (LSD) test (homogeneity of variance) or the Games-Howell test (non-homogeneity of variance), was used to analyze differences between groups. Neurobehavioral scores were analyzed with non-parametric tests (Kruskal-Wallis tests, followed by Mann-Whitney U tests for comparison between groups). Kaplan-Meier curves and log-rank tests were employed for survival analysis. $\mathrm{P}<0.05$ was considered to indicate a statistically significant difference.

\section{Results}

JNK1 siRNA downregulates JNK1 transcription in rats. At $24 \mathrm{~h}$ following the in vivo injection of the transfected reagents, the RT-qPCR results (Fig. 1) revealed that the JNK1 transcript level in the siRNA group was significantly decreased compared with that in the scramble group $(\mathrm{F}=279.36$; $\mathrm{P}<0.001)$, which indicated that the in vivo RNAi rat model had been successfully established.

JNK1 inhibition improves the neuro-rehabilitation and survival times of rats. The SAH rat models were successfully constructed as previously described by Zhang et al (20). The inferior basal temporal lobe was stained with blood, indicating that the adjacent brain tissue, in particular the hippocampus, may be injured by SAH-induced inflammatory responses (Fig. 2). To evaluate the effect of JNK1 inhibition on neuroprotection, the mean neurodeficit scores of 6 randomly chosen rats in each group were recorded and analyzed. In addition, survival analyses were also performed. The results demonstrated that there were significant differences in neurological scores among the groups (Kruskal-Wallis $\chi^{2}=24.198, \mathrm{P}<0.001$; Fig. 3). The mean score at $72 \mathrm{~h}$ was significantly decreased compared with 
Table I. Sequences of primers in reverse transcription quantitative polymerase chain reaction.

\begin{tabular}{|c|c|c|c|c|}
\hline Genes & Sequences & Primer, bp & $\mathrm{Tm},{ }^{\circ} \mathrm{C}$ & Products, bp \\
\hline JNK1 F & AGTTATTGAACAGCTCGGAA & 20 & 52.6 & 217 \\
\hline JNK1 R & TTTGGACGCATCTATCACC & 19 & 53.7 & \\
\hline p53 F & CAGAGTTGTTAGAAGGCCCAGAG & 23 & 60.2 & 136 \\
\hline p53 R & TGAGAAGGGACGGAAGATGAC & 21 & 58.9 & \\
\hline Bax F & TCCACCAAGAAGCTGAGCGAG & 21 & 65.2 & 98 \\
\hline Bax R & GTCCAGGCCCATGATGGTTCT & 21 & 65.6 & \\
\hline Bcl-2 F & TGAACCGGCATCTGCACAC & 19 & 64.4 & 96 \\
\hline Bcl-2 R & CGTCTTCAGAGACAGCCAGGAG & 22 & 63.8 & \\
\hline p38 F & CGGCTTGCTCATGTCCTCAGAAC & 22 & 67.6 & 214 \\
\hline p38 R & GGAGGGCGGCTGCACATACAC & 21 & 69.3 & \\
\hline $\mathrm{NF}-\kappa \mathrm{B} F$ & ACGATCTGTTTCCCCTCATC & 20 & 58.9 & 241 \\
\hline$N F-\kappa B R$ & TGCTTCTCTCCCCAGGAATA & 20 & 59.8 & \\
\hline Caspase-3 F & GACGACAGGGTGCTACGAT & 22 & 56.9 & 193 \\
\hline Caspase-3 R & TTTCCTTACGCTCTGACTGA & 19 & 55.6 & \\
\hline$\beta$-actin F & GGAGATTACTGCCCTGGCTCCTAGC & 25 & 60.1 & 155 \\
\hline$\beta$-actin $\mathrm{R}$ & GGCCGGACTCATCGTACTCCTGCTT & 25 & 62.0 & \\
\hline
\end{tabular}

F, forward; R, reverse; JNK1, c-Jun N-terminal kinase 1; p53, tumor protein p53; Bcl-2, B-cell lymphoma 2; Bax, Bcl-2-associated X protein; p38, mitogen-activated protein kinase $11 ; \mathrm{NF}-\kappa \mathrm{B}$, nuclear factor $\kappa$-light-chain-enhancer of activated B-cells; Tm, melting temperature.

Table II. Behavior and activity scores.

\begin{tabular}{llc}
\hline Category & \multicolumn{1}{c}{ Behavior } & Score \\
\hline Appetite & Finished meal & 0 \\
& Left meal unfinished & 1 \\
& Scarcely ate & 2 \\
Activity & Walked and reached at least & 0 \\
& three corners of the cage & \\
& Walked with some stimulations & 1 \\
& Almost always lying down & 2 \\
Motor deficit & No deficit & 0 \\
& Unstable walk & 1 \\
& Impossible to walk & 2 \\
\hline
\end{tabular}

that at $24 \mathrm{~h}$ following SAH in the siRNA group $(\mathrm{P}=0.003)$. By contrast, in the scramble group, the mean score at $24 \mathrm{~h}$ did not reveal any significant difference compared with that at $72 \mathrm{~h}(\mathrm{P}=0.118)$. At $24 \mathrm{~h}$ following $\mathrm{SAH}$, the scramble and the siRNA groups exhibited similar mean scores $(\mathrm{P}=0.206)$. At $72 \mathrm{~h}$, the mean score in the siRNA group was significantly decreased compared with that in the scramble group $(\mathrm{P}=0.003)$. Kaplan-Meier curve analysis revealed that the median survival time in the siRNA group was significantly increased compared with that in the scramble group (log-rank $\mathrm{P}=0.038$ ).

JNK1 downregulation decreases the level of cell apoptosis in the hippocampus. Hippocampus tissues were harvested for pathological analysis. TUNEL assays were employed to detect the rates of apoptosis. The results of the TUNEL assay (Fig. 4) demonstrated that the apoptosis ratio in the scramble $72 \mathrm{~h}$ group
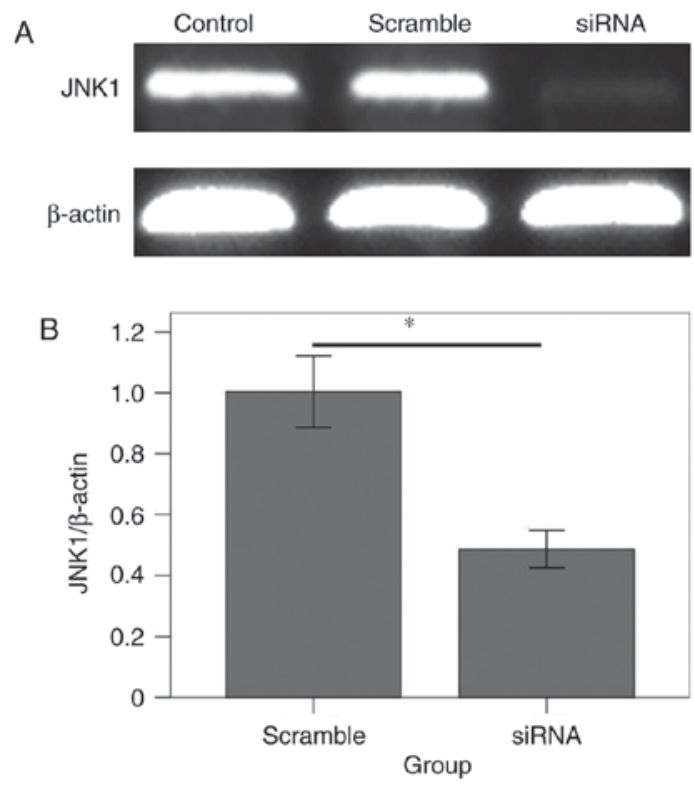

Figure 1. (A) JNK1 transcript levels in the three groups of transfected rat hippocampus tissues, as determined by reverse transcription quantitative polymerase chain reaction. (B) The JNK1 transcript levels in the siRNA group were significantly decreased compared with those in the scramble group. ${ }^{*} \mathrm{P}<0.05$. JNK1, c-Jun terminal kinase 1; siRNA, small interfering RNA.

was significantly increased compared with that in the scramble $24 \mathrm{~h}$ group $(\mathrm{P}<0.001)$. However, in the siRNA group, the apoptosis ratio at $72 \mathrm{~h}$ after $\mathrm{SAH}$ was almost at an identical level with the ratio at $24 \mathrm{~h}$ after $\mathrm{SAH}(\mathrm{P}=0.963)$. At 24 and $72 \mathrm{~h}$, the apoptosis ratio in the RNAi group was significantly decreased compared with that in the scramble group (after $24 \mathrm{~h}, \mathrm{P}=0.010$; after $72 \mathrm{~h}, \mathrm{P}<0.001)$. Nissl staining was subsequently performed 


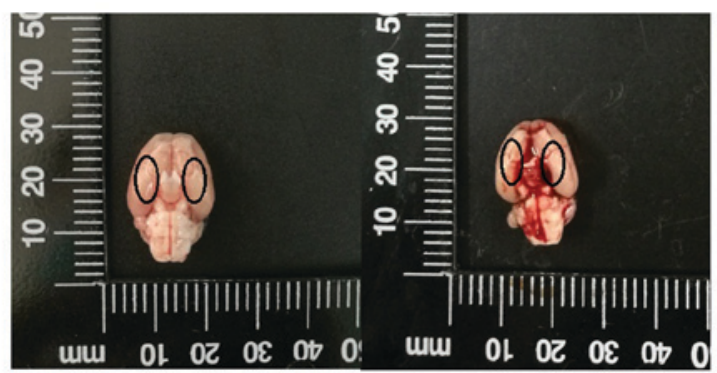

Figure 2. Representative images of rat brain tissue. (Left image) In the sham-operated group, no blood was identified on the surface of the brain. (Right image) In the subarachnoid hemorrhage group, the inferior basal temporal lobe was stained with blood. The hippocampus areas of brain (depicted by the area of black ovals, which were most sensitive to apoptotic stimulation) were obtained for subsequent analysis.

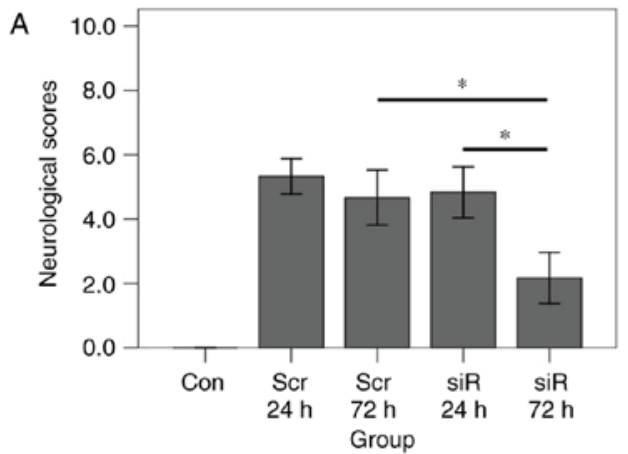

B

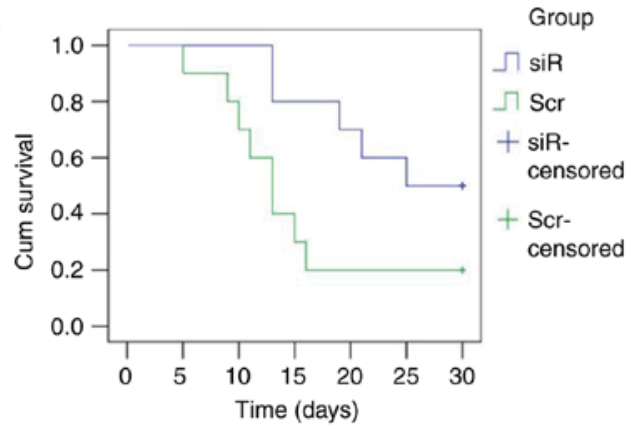

Figure 3. Neurological scores and survival curve analysis of SAH rats following the different treatments. (A) The neurological scores assay revealed that the siR group exhibited a significantly decreased score compared with the Scr group at $72 \mathrm{~h}$ following SAH. The score of the siR group at $72 \mathrm{~h}$ was also decreased compared with that at $24 \mathrm{~h}$. (B) The survival assay revealed that the average survival time of rats in the siR group was significantly increased compared with that in the Scr group. SAH, subarachnoid hemorrhage. "P<0.05. Con, control; siR, siRNA; Scr, scramble.

to reveal the numbers of intact neurons in the hippocampus area. As demonstrated in Fig. 5, at 24 and 72 h following SAH, the number of intact neurons in the RNAi group was significantly increased compared with that in the scramble group $(\mathrm{P}<0.001)$. In the scramble group, the number of neurons at $72 \mathrm{~h}$ following SAH was markedly decreased compared with the number present at $24 \mathrm{~h}$ after SAH $(\mathrm{P}<0.001)$. By contrast, in the RNAi group, the numbers of living neurons at the 2 time points were at an almost identical level $(\mathrm{P}=0.220)$.

JNK1 RNAi downregulates the expression levels of apoptosis-associated genes. To investigate the biological mechanism of decreased apoptosis levels via JNK1 gene silencing, RT-qPCR and western blot analysis assays were performed. The results of the RT-qPCR revealed that, at 24 and $72 \mathrm{~h}$, the JNK1 transcript levels in the RNAi group were significantly decreased compared with those in the control group (at 24 h, $\mathrm{P}<0.001$; at 72 h, $\mathrm{P}<0.001$; Fig. 6). By contrast, the JNK1 transcript levels in the scramble group were at an almost identical level compared with those in the control group (at $24 \mathrm{~h}, \mathrm{P}=0.500$; at $72 \mathrm{~h}, \mathrm{P}=0.757$ ). At the 2 time points, the JNK1 transcript level in the RNAi group was decreased compared with that in the scramble group (at $24 \mathrm{~h}$, $\mathrm{P}<0.001$; at $72 \mathrm{~h}, \mathrm{P}<0.001)$. The p53 transcript levels in all groups were similar $(\mathrm{P}=0.398)$. At $24 \mathrm{~h}$, the Bax transcript levels in the scramble and RNAi groups were significantly increased compared with that in the control group (scramble group, $\mathrm{P}<0.001$; RNAi group, $\mathrm{P}<0.001$ ); the Bax transcript level in the scramble group was also significantly increased compared with that in the RNAi group $(\mathrm{P}<0.001)$. After $72 \mathrm{~h}$, the Bax transcript level in the scramble group was significantly increased compared with that in the control group $(\mathrm{P}<0.001)$, although the transcript level in the RNAi group was almost identical with that in the control group $(\mathrm{P}=0.771)$; in addition, the Bax transcript level in the scramble group was significantly increased compared with that in the RNAi group $(\mathrm{P}<0.001)$. In the RNAi group, the Bcl-2 transcript levels at 24 and $72 \mathrm{~h}$ were significantly increased compared with that in the control group ( $\mathrm{P}<0.001$ for the 2 time points). The Bcl-2 transcript level in the scramble group at $24 \mathrm{~h}$ was significantly decreased compared with that in the control group $(\mathrm{P}<0.001)$, but the transcript level at $72 \mathrm{~h}$ was almost identical with that of the control group $(\mathrm{P}=0.074)$. At $24 \mathrm{~h}$, the $\mathrm{Bcl}-2$ transcript level in the RNAi group was significantly increased compared with that in the scramble group $(\mathrm{P}<0.001)$. At $24 \mathrm{~h}$, the $\mathrm{p} 38$ transcript levels in the scramble and the RNAi groups were significantly increased compared with that in the control group $(\mathrm{P}<0.001)$. At $72 \mathrm{~h}$, the p38 transcript level in the scramble and the RNAi groups were significantly increased compared with that in the control group $(\mathrm{P}<0.001)$. At the 2 time points $(24$ or $72 \mathrm{~h})$, the p38 transcript levels in the RNAi group was almost identical with that of the scramble group (at $24 \mathrm{~h}, \mathrm{P}=0.825$; at $72 \mathrm{~h}$, $\mathrm{P}=0.546$ ). At $24 \mathrm{~h}$, the $\mathrm{NF}-\kappa \mathrm{B}$ transcript levels in the scramble and the RNAi groups were significantly increased compared with that in the control group $(\mathrm{P}<0.001)$. At $72 \mathrm{~h}$, the $\mathrm{NF}-\kappa \mathrm{B}$ transcript levels in the scramble and the RNAi groups were significantly increased compared with that in the control group (scramble group: $\mathrm{P}=0.001$; RNAi group, $\mathrm{P}<0.001$ ). Irrespective of whether the data at 24 or $72 \mathrm{~h}$ were being compared, the $\mathrm{NF}-\kappa \mathrm{B}$ transcript level in the RNAi group was almost at the same level as that of the scramble group (at $24 \mathrm{~h}, \mathrm{P}=0.739$; at $72 \mathrm{~h}, \mathrm{P}=0.469$ ). Finally, at $24 \mathrm{~h}$ the caspase- 3 transcript level in the scramble and the RNAi groups were significantly increased compared with that in the control group $(\mathrm{P}<0.001)$. At $72 \mathrm{~h}$, the caspase- 3 transcript levels in the scramble and the RNAi groups were significantly increased compared with that in the control group $(\mathrm{P}<0.001)$. At the 2 time points $(24$ or $72 \mathrm{~h}$ ), the caspase- 3 transcript level in the RNAi group was at a very similar level to that of the scramble group (at $24 \mathrm{~h}$, $\mathrm{P}=0.534$; at $72 \mathrm{~h}, \mathrm{P}=0.345$ ).

Representative bands and correlated histograms of the western blot analysis experiments are presented in Fig. 7. 


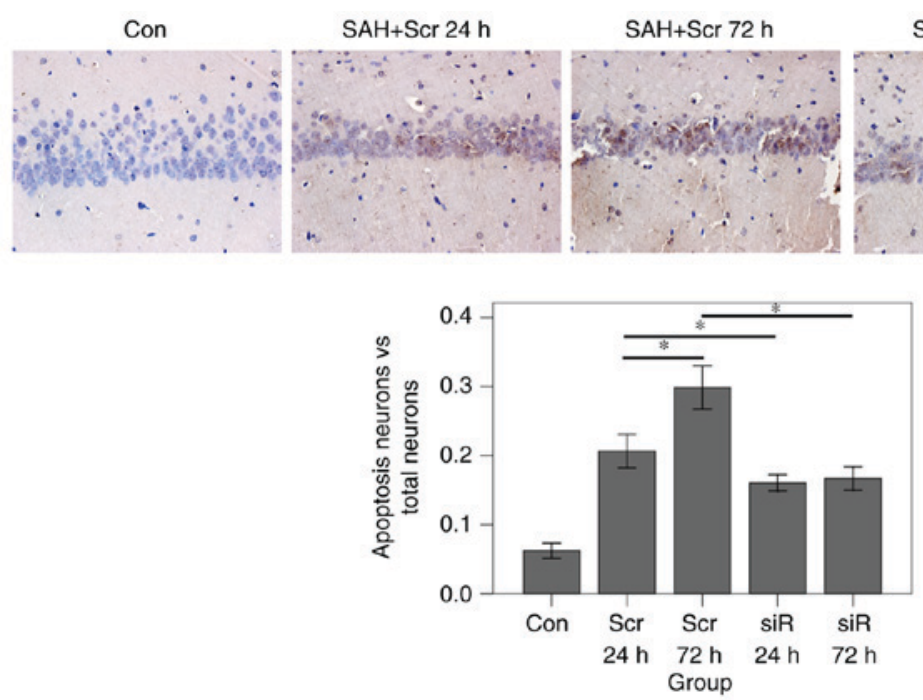

Figure 4. Terminal deoxynucleotidyl-transferase-mediated dUTP nick-end labeling assays revealed the apoptotic neurons in the hippocampus areas of rat brains. Images are at magnification, $x 400$. The apoptotic rate in the Scr group was significantly increased compared with that in the siR group at the same time point. JNK1 inhibition resulted in an inhibition of the increasing apoptotic rate observed between 24 and $72 \mathrm{~h}$. " $\mathrm{P}<0.05$. Con, control; siR, siRNA; Scr, scramble; SAH, subarachnoid hemorrhage.

A Con $\mathrm{SAH}+\mathrm{Scr} 24 \mathrm{~h}$ $\mathrm{SAH}+\mathrm{Scr} 72 \mathrm{~h}$ $\mathrm{SAH}+\mathrm{SiR} 24 \mathrm{~h}$ $\mathrm{SAH}+\mathrm{SiR} 72 \mathrm{~h}$
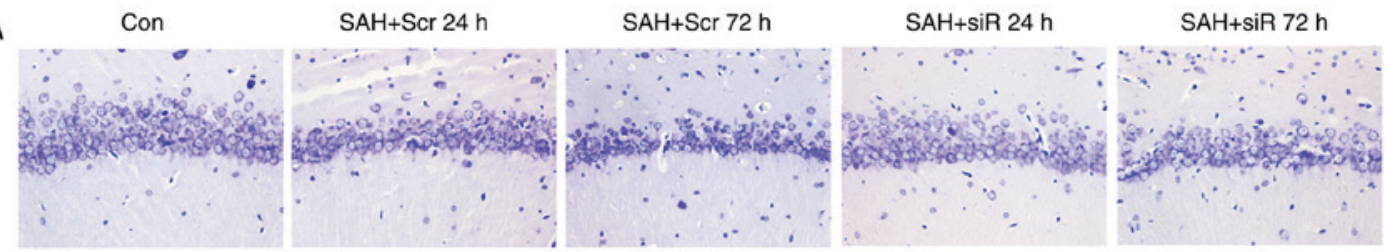

B

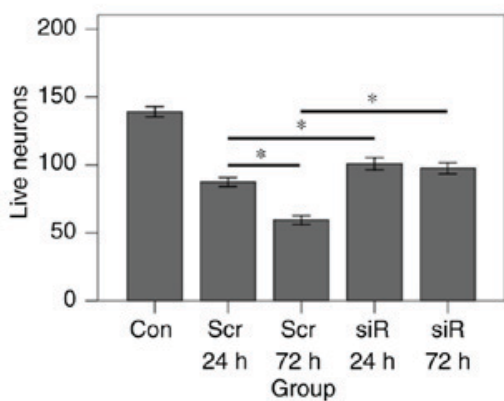

Figure 5. Nissl staining of the hippocampus areas of rat brains reveal the living neurons. (A) Images are at magnification, $\mathrm{x} 400$. (B) The number of living neurons in the siR group was significantly increased compared with that in the Scr group at the 24 and $72 \mathrm{~h}$ time points following SAH. JNK1 inhibition inhibited continuously death of the neurons between 24 and 72 h. "P<0.05. JNK1, c-Jun terminal kinase 1. Con, control; siR, siRNA; Scr, scramble; SAH, subarachnoid hemorrhage.

The p-JNK expression levels in the scramble group after 24 $(\mathrm{P}<0.001)$ and $72 \mathrm{~h}(\mathrm{P}=0.009)$ were significantly increased compared with that in the control group. The levels of the same protein in the RNAi group at $24(\mathrm{P}=0.008)$ and $72 \mathrm{~h}$ $(\mathrm{P}=0.001)$ were significantly decreased compared with that in the control group. At 24 and $72 \mathrm{~h}$, the p-JNK expression levels in the RNAi group were significantly decreased compared with that in the scramble group $(\mathrm{P}<0.001$ for the 2 time points). The p-p53 expression levels in the scramble $24 \mathrm{~h}$ $(\mathrm{P}<0.001)$ and scramble $72 \mathrm{~h}(\mathrm{P}=0.009)$ groups were significantly increased compared with that in the control group. The expression levels of the same protein in the RNAi $24 \mathrm{~h}$ $(\mathrm{P}=0.001)$ and RNAi $72 \mathrm{~h}(\mathrm{P}=0.001)$ groups were significantly decreased compared with that in the control group. At 24 and
$72 \mathrm{~h}$, the p-p53 expression levels in the RNAi group were significantly decreased compared with that in the scramble group ( $\mathrm{P}<0.001$ for the 2 time points). The Bax protein expression levels in the scramble $24 \mathrm{~h}$, scramble $72 \mathrm{~h}$, and RNAi 24 h groups were all significantly increased compared with that in the control group (all $\mathrm{P}<0.001$ ). However, the expression level of the same protein in the RNAi $72 \mathrm{~h}$ group was not significantly different from that in the control group $(\mathrm{P}=0.268)$. At 24 and $72 \mathrm{~h}$, the Bax expression levels in the RNAi group were significantly decreased compared with that in the scramble group ( $\mathrm{P}<0.001$ for the 2 time points). The Bcl-2 protein expression levels in the scramble $72 \mathrm{~h}$, the RNAi $24 \mathrm{~h}$, and the RNAi $72 \mathrm{~h}$ groups were all significantly increased compared with that in the control group (all 

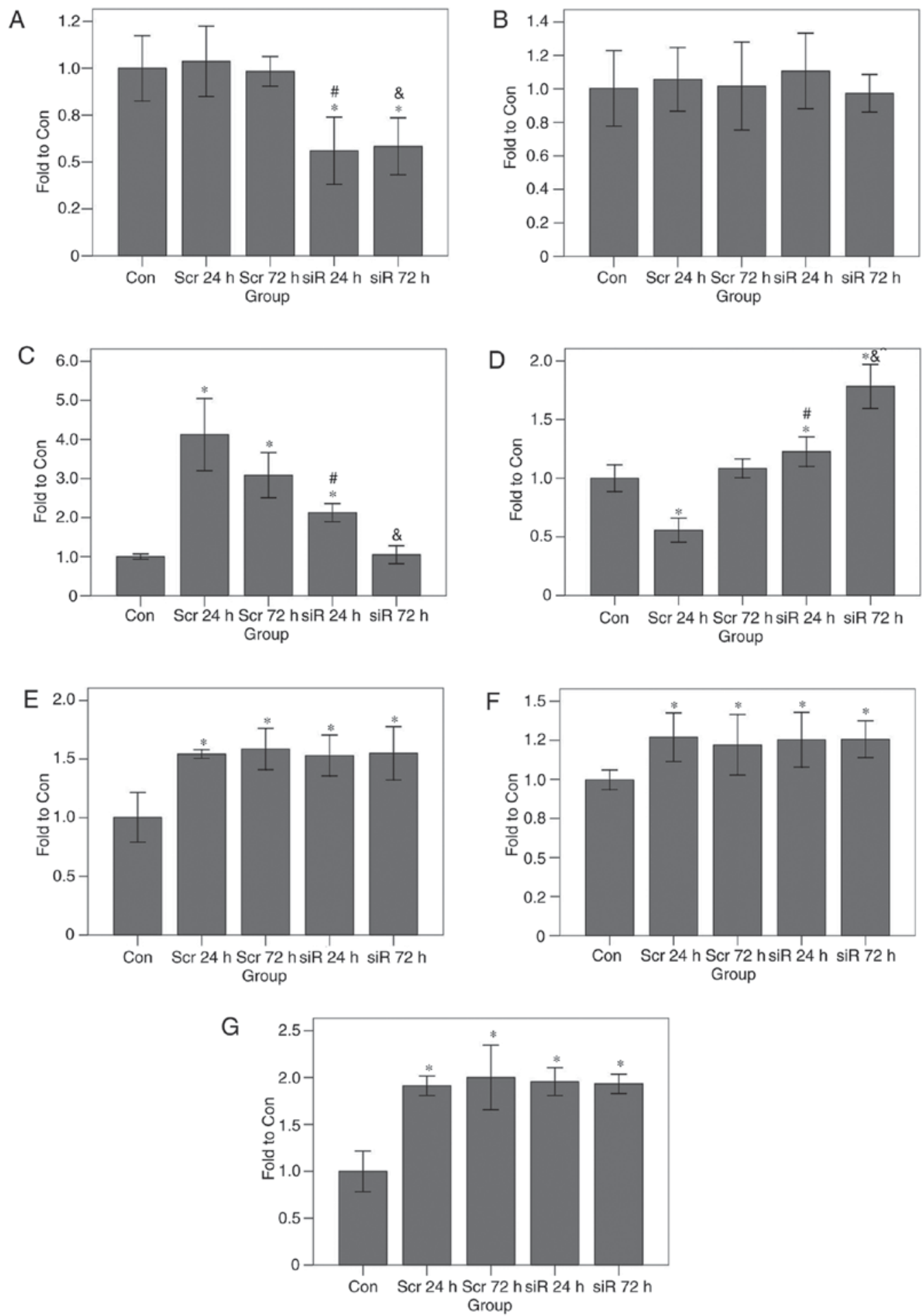

Figure 6. Effects of JNK1 inhibition on transcription levels of apoptosis-associated genes as determined by reverse transcription quantitative polymerase chain reaction. JNK1 inhibition led to an increase in the Bcl-2 transcript level and a decrease in the Bax transcript level, but no significant effect on any other genes was observed. The transcript levels for (A) c-Jun terminal kinase 1, (B) tumor protein 53, (C) Bax, (D) Bcl-2, (E) mitogen-activated protein kinase 11, (F) nuclear factor $\kappa$-light-chain-enhancer of activated B-cells, and $(\mathrm{G})$ caspase-3 are presented. "P $<0.05$ vs. Con group; ${ }^{\#} \mathrm{P}<0.05$ vs. the Scr $24 \mathrm{~h}$ group; ${ }^{\&} \mathrm{P}<0.05$ vs. the Scr 72 h group. Bcl-2, B-cell lymphoma 2; Bax, Bcl-2-associated X protein; Con, control; siR, siRNA; Scr, scramble.

$\mathrm{P}<0.001)$. The expression level of the same protein in the scramble 24 h group was significantly decreased compared with that in the control group $(\mathrm{P}<0.001)$. At 24 and $72 \mathrm{~h}$, the Bax expression levels in the RNAi group were significantly increased compared with that in the scramble group (both $\mathrm{P}<0.001)$. The $\mathrm{p}-\mathrm{p} 38$ protein expression levels in the scramble $24 \mathrm{~h}$, the scramble $72 \mathrm{~h}$, the RNAi $24 \mathrm{~h}$ and the RNAi $72 \mathrm{~h}$ groups were all significantly increased compared with that in the control group (all $\mathrm{P}<0.001$ ). No significant differences were identified among the four experimental groups $(\mathrm{F}=1.771$; $\mathrm{P}=0.230)$. The $\mathrm{NF}-\kappa \mathrm{B}-\mathrm{p} 65$ expression levels in the scramble $24 \mathrm{~h}(\mathrm{P}=0.019)$, the scramble $72 \mathrm{~h}(\mathrm{P}=0.023)$, the RNAi $24 \mathrm{~h}$ $(\mathrm{P}=0.004)$, and the RNAi $72 \mathrm{~h}(\mathrm{P}=0.001)$ groups were all significantly increased compared with that in the control 
A

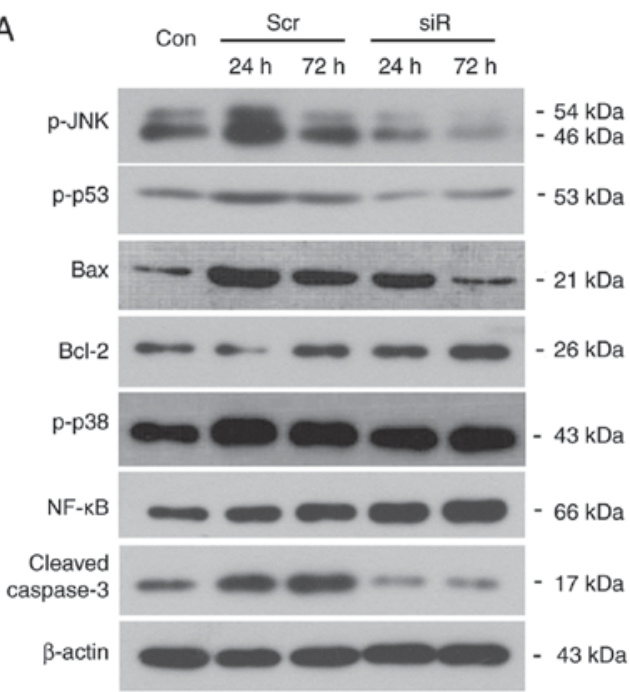

C

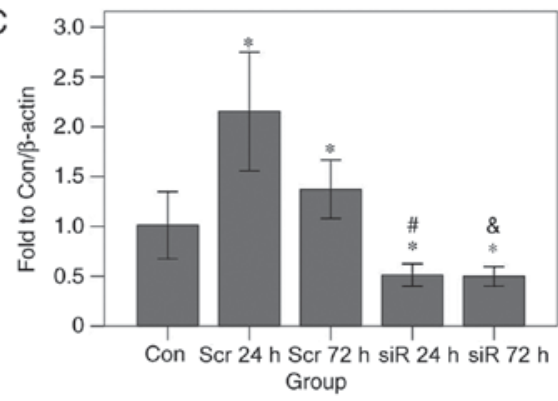

E
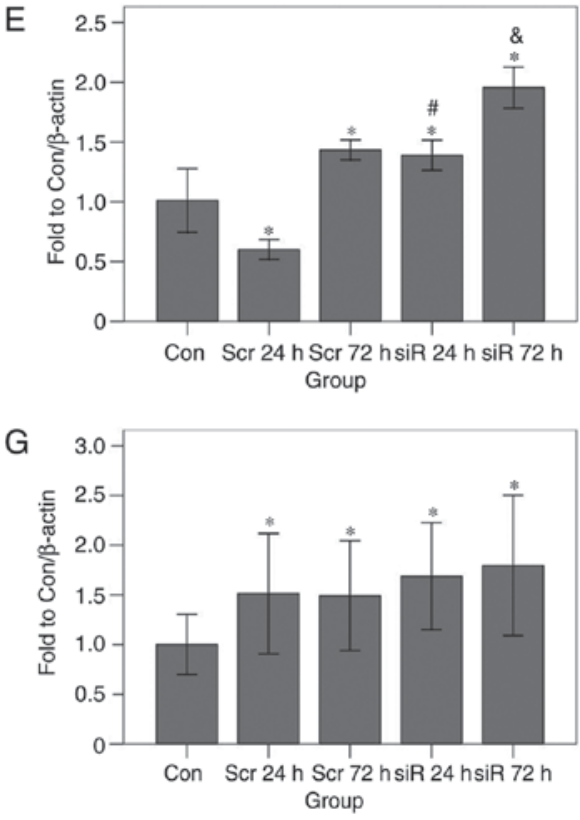
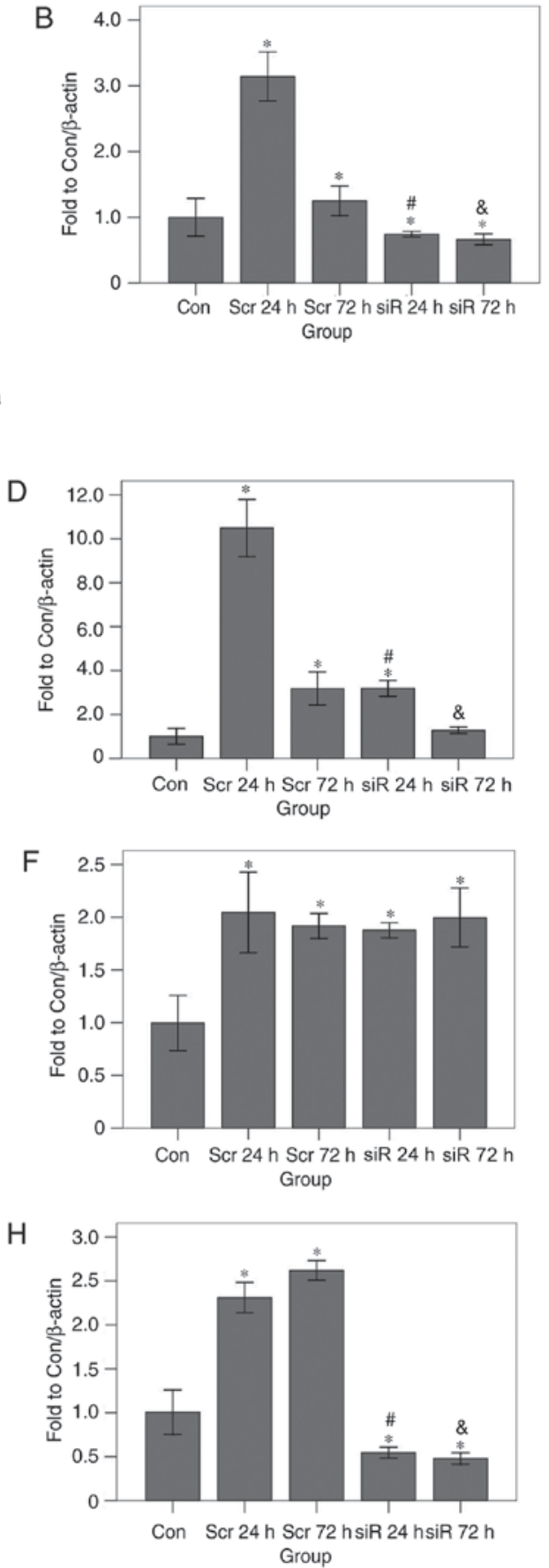

Figure 7. Effects of JNK1 inhibition on the protein expression levels of apoptosis-associated genes as determined by western blot analysis. (A) Representative bands are presented. JNK1 inhibition led to an increase in Bcl-2 expression and a decrease in the protein expression levels of p-p53, Bax and cleaved

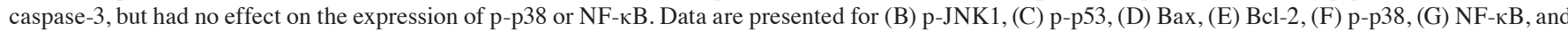
(H) Cleaved caspase-3. ${ }^{~} \mathrm{P}<0.05$ vs. the Con group, ${ }^{\text {"P }} \mathrm{P}<0.05$ vs. the $\mathrm{Scr} 24 \mathrm{~h}$ group; ${ }^{\circledR} \mathrm{P}<0.05$ vs. the $\mathrm{Scr} 72 \mathrm{~h}$ group. p, phosphorylated; p38, mitogen-activated protein kinase 11; p53, tumor protein 53; JNK1, c-Jun terminal kinase 1; Bcl-2, B-cell lymphoma 2; Bax, Bcl-2-associated X protein; NF-kB, nuclear factor k-light-chain-enhancer of activated B-cells; Con, control; siR, siRNA; Scr, scramble.

group. Again, no significant differences were identified among the four experimental groups $(\mathrm{F}=1.042, \mathrm{P}=0.412)$. Finally, at 24 and $72 \mathrm{~h}$ the cleaved caspase-3 protein expression levels in the scramble group were significantly increased compared with that in the control group ( $\mathrm{P}=0.001$ for the 2 time points). The expression levels of the same protein in the RNAi $24 \mathrm{~h}$ $(\mathrm{P}=0.040)$ and the RNAi $72 \mathrm{~h}(\mathrm{P}=0.029)$ groups were significantly decreased compared with that in the control group. 
At 24 and $72 \mathrm{~h}$, the cleaved caspase-3 expression level in the RNAi group was significantly decreased compared with that in the scramble group ( $\mathrm{P}<0.001$ for the two time points). In the scramble group, the cleaved caspase- 3 level at $72 \mathrm{~h}$ was significantly increased compared with that at $24 \mathrm{~h}(\mathrm{P}=0.021)$.

\section{Discussion}

In the present study, the mRNA transcript of JNK1 was first downregulated in an aSAH rat model, and any ethological and histological variations were subsequently investigated. The period from 24 to $72 \mathrm{~h}$ following SAH is considered to be an important phase for EBI, during which SAH induces a prominent impairment of the clinical behavioral function (20). The present study demonstrated that JNK1 inhibition attenuated the neurodeficit scores of SAH rats at $72 \mathrm{~h}$. Additionally, TUNEL assays revealed that JNK1 inhibition reversed the increase in levels of apoptotic cells in the hippocampus. According to the Nissl staining results, it was also identified that the RNAi group exhibited a markedly increased number of living neurons compared with the scramble group at 24 and $72 \mathrm{~h}$ following SAH. In the RNAi group, the number of living neurons at $72 \mathrm{~h}$ were not markedly decreased compared with that at $24 \mathrm{~h}$, indicating that neuron apoptosis had been interrupted. Consequently, we hypothesized that the quick recovery of neurological function at $72 \mathrm{~h}$ in the RNAi group had resulted from the interruption of cascaded cell apoptosis. In the majority of cases, neuronal cell death due to aSAH would be experienced as a hypoxic process caused by numerous pathological events, including blood-brain barrier dysfunction, edema, inflammation and oxidative cascades (21). Thereafter, it was inferred that the downregulation of JNK1 may strengthen the hypoxic endurance of the neurons. These results are comparable with those from a study comprising a JNK1 inhibitor that led to an improvement in the survival time of neurons in an EBI model via the suppression of Nur77-dependent apoptosis pathways (15). However, that study lacked data associated with any effects observable $24 \mathrm{~h}$ following SAH. The present study has provided additional information regarding the protective effects of JNK1 inhibition on EBI at $72 \mathrm{~h}$ following SAH. Under the identical conditions, the survival times of the SAH rats were also recorded. Upon JNK1 inhibition, a clear improvement in the survival times of the SAH rats was observed, indicating that the interruption of the EBI process may improve the resistance of neurons to subsequent effects of SAH.

To elucidate the molecular mechanism, RT-qPCR and western blot analyses were performed. The results revealed that $\mathrm{SAH}$ led to an increase in the transcription and protein expression levels of the phosphorylated proteins of JNK1, p38 and NF- $\kappa$ B, which are all associated with promoting pro-apoptotic and pro-inflammatory cellular signaling pathways, leading to enhanced SAH-induced inflammatory responses and poorer SAH outcomes $(22,23)$. It has been demonstrated that decreasing the activation of these pathways simultaneously with a specific transforming growth factor $\beta$-activated kinase 1 inhibitor, 5Z-7-oxozeaenol, effectively prevented neuronal apoptosis and attenuated neurological deficits in an SAH model (20). However, the effects of inhibiting the pathways separately has not been described extensively. In the present study, it was demonstrated that JNK1 inhibition did not affect the phosphorylation of $\mathrm{p} 38$ and $\mathrm{NF}-\kappa \mathrm{B}$. It was suggested that the JNK1 signaling pathway may be independent of the other 2 pathways in the SAH model, rather than JNK1 being positioned downstream of them, as has been suggested by certain studies $(24,25)$. Additional studies are required to gain novel information concerning the detailed interactions among the 3 pathways. p53 has emerged as one of the most important anti-apoptosis targets in SAH (26). The present study demonstrated that the expression of p-p53 was synchronized with that of p-JNK1, although transcription of the p53 gene was not affected by JNK1 inhibition, indicating that JNK1 and p53 interact at the level of phosphorylation, a result that is consistent with the mechanisms operating in oxidative stress-induced apoptosis (13). Once p53 signaling is inhibited, at least one apoptotic pathway will be blocked. The downregulation of Bax protein and upregulation of Bcl-2 observed in the present study suggested deactivation of the mitochondrial apoptotic pathway, accompanied by the downregulation of cleaved caspase-3. As mentioned above, the levels of cleaved caspase-3 in the RNAi group were significantly decreased compared with that in the scramble group at the same time point. Caspase-3 may be cleaved either via the intrinsic apoptotic pathway (mitochondrial pathway) or via the extrinsic apoptotic pathway (death receptor pathway) (27). Based on the results in the present study, it may be possible that JNK1 inhibition prevented the mitochondrial apoptotic pathway from exerting a role in the overall EBI process.

The association between JNK and p53 has previously been investigated in a model of DNA damage caused by cisplatin, a chemotherapeutic drug used for cancer treatment (28). In the process of cisplatin-induced DNA damage, p53 protein levels accumulated gradually, and may have been regulated by JNK. It was suggested that JNK1 functions as a negative regulator in the process (13). Furthermore, an additional study investigating signaling in a model of fibronectin fragment-mediated apoptosis verified this conclusion (29). However, in the present study, the opposite phenomenon was observed, suggesting that JNK1 may interact with p53 differently according to the prevailing circumstances. In fact, a study examining deoxycholic acid-induced liver cell apoptosis also revealed that JNK may directly or indirectly modulate p53 expression and positively affect apoptosis. The possible mechanism suggested was of p53 stabilization and its increased ability to elicit apoptosis, which resulted from JNK-induced p53 phosphorylation and attenuation of the interaction of p53 with mouse double minute 2 homolog $(28,30)$.

Even though a putative preliminary mechanism was suggested by the results from the present study, there were certain limitations. Although the method of pre-chiasmatic cistern injection provided a reasonably homogenous animal model, it was not fully able to simulate the in vivo situation of aSAH, as the intracranial closeness was broken. The method of internal carotid artery puncture may provide a closer approximation to the in vivo conditions of aSAH, according to previous studies $(31,32)$; however, in the present study the quantity of hemorrhage and severity of subsequent complications would not be easy to maintain at the same level. Consequently, these 2 approaches will continue to be employed according to the preferences of the specific study, until improved methods are devised. 
In conclusion, in the present study the expression of JNK1 was downregulated with in vivo RNAi technology, which revealed that JNK1 inhibition improves the neurological scores and survival times of SAH rats. The mechanism that has been proposed involves an interruption of neuronal apoptosis in the EBI process. JNK1 inhibition may contribute to this process by decreasing p53 phosphorylation and deactivating the downstream mitochondrial apoptotic pathway.

\section{Acknowledgements}

Not applicable.

\section{Funding}

The present study was supported by Special funds for Scientific and Technological Innovation Talents Research from Harbin Science and Technology Bureau (grant no. 2014RFXGJ015) and the National Natural Science Foundation of China (grant no. 81302177).

\section{Availability of data and materials}

The datasets used and analyzed during the current study are available from the corresponding author on reasonable request.

\section{Authors' contributions}

All authors participated in the design, interpretation of the studies and analysis of the data and review of the manuscript. GQL, XFL and WY designed the protocol of experiments. XHL constructed the aSAH animal model and conducted the in vivo transfection protocol. ZYW collected the brain tissue and conducted the TUNEL assays and Nissl staining. DYM conducted the RT-qPCR and western blot analysis assays. YW recorded the behavioral scores and survival data. GQL and WY wrote the manuscript. XFL performed the statistical analysis.

\section{Ethics approval and consent to participate}

All procedures were approved by Harbin Medical University Animal Care and Use Committee and in accordance with the Guide for the Care and Use of Laboratory Animals by the National Institute of Health.

\section{Patient consent for publication}

Not applicable.

\section{Competing interests}

The authors declare that they have no competing interests.

\section{References}

1. Danière F, Gascou G, Menjot de Champfleur N, Machi P Leboucq N, Riquelme C, Ruiz C, Bonafé A and Costalat V: Complications and follow up of subarachnoid hemorrhages Diagn Interv Imaging 96: 677-686, 2015.

2. Macdonald RL, Pluta RM and Zhang JH: Cerebral vasospasm after subarachnoid hemorrhage: The emerging revolution. Nat Clin Pract Neurol 3: 256-263, 2007.
3. Macdonald RL, Higashida RT, Keller E, Mayer SA, Molyneux A, Raabe A, Vajkoczy P, Wanke I, Bach D, Frey A, et al: Clazosentan, an endothelin receptor antagonist, in patients with aneurysmal subarachnoid haemorrhage undergoing surgical clipping: A randomised, double-blind, placebo-controlled phase 3 trial (CONSCIOUS-2). Lancet Neurol 10: 618-625, 2011.

4. Sehba FA, Hou J, Pluta RM and Zhang JH: The importance of early brain injury after subarachnoid hemorrhage. Prog Neurobiol 97: 14-37, 2012.

5. Yuksel S, Tosun YB, Cahill J and Solaroglu I: Early brain injury following aneurysmal subarachnoid hemorrhage: Emphasis on cellular apoptosis. Turk Neurosurg 22: 529-533, 2012.

6. Hasegawa Y, Suzuki H, Sozen T, Altay O and Zhang JH: Apoptotic mechanisms for neuronal cells in early brain injury after subarachnoid hemorrhage. Acta Neurochir Suppl 110: 43-48, 2011.

7. Cahill J, Calvert JW and Zhang JH: Mechanisms of early brain injury after subarachnoid hemorrhage. J Cereb Blood Flow Metab 26: 1341-1353. 2006.

8. Zhou C, Yamaguchi M, Colohan AR and Zhang JH: Role of p53 and apoptosis in cerebral vasospasm after experimental subarachnoid hemorrhage. J Cereb Blood Flow Metab 25: 72-82, 2005.

9. Dawson VL and Dawson TM: Deadly conversations: Nuclear-mitochondrial cross-talk. J Bioenerg Biomembr 36: 287-294, 2004.

10. van Loo G, Saelens X, van Gurp M, MacFarlane M, Martin SJ and Vandenabeele P: The role of mitochondrial factors in apoptosis: A Russian roulette with more than one bullet. Cell Death Differ 9: 1031-1042, 2002.

11. Dérijard B, Hibi M, Wu IH, Barrett T, Su B, Deng T, Karin M and Davis RJ: JNK1: A protein kinase stimulated by UV light and Ha-Ras that binds and phosphorylates the c-Jun activation domain. Cell 76: 1025-1037, 1994.

12. Gkouveris I and Nikitakis NG: Role of JNK1 signaling in oral cancer: A mini review. Tumour Biol 39: 1010428317711659, 2017.

13. Fuchs SY, Adler V, Pincus MR and Ranai Z: MEKK1/JNK signaling stabilizes and activates p53. Proc Natl Acad Sci USA 95: 10541-10546, 1998.

14. Ferreira DM, Afonso MB, Rodrigues PM, Simão AL, Pereira DM, Borralho PM, Rodrigues CM and Castro RE: c-Jun N-terminal kinase 1/c-Jun activation of the p53/MicroRNA 34a/Sirtuin 1 pathway contributes to apoptosis induced by deoxycholic acid in rat liver. Mol Cell Biol 34: 1100-1120, 2014.

15. Dai YX, Zhang W, Zhou XM and Shi J: Inhibition of c-Jun $\mathrm{N}$-terminal kinase ameliorates early brain injury after subaracnoid hemorrhage through inhibition of a Nur77 dependent pathway. Neurochem Res 39: 1603-1611, 2014.

16. National Research Council (US) Committee for the Update of the Guide for the Care and Use of Laboratory Animals: Guide for the Care and Use of Laboratory Animals. National Academies Press, Washington, DC, 2010.

17. Shen H, Chen Z, Wang Y, Gao A, Li H, Cui Y, Zhang L, Xu X, Wang $Z$ and Chen G: Role of neurexin- $1 \beta$ and neuroligin-1 in cognitive dysfunction after subarachnoid hemorrhage in rats. Stroke 46: 2607-2615, 2015

18. Livak KJ and Schmittgen TD: Analysis of relative gene expression data using real-time quantitative PCR and the 2(-Delta Delta C(T)) method. Methods 25: 402-408, 2001.

19. Wang Z, Ma C, Meng CJ, Zhu GQ, Sun XB, Huo L, Zhang J, Liu HX, He WC, Shen XM, et al: Melatonin activates the Nrf2-ARE pathway when it protects against early brain injury in a subarachnoid hemorrhage model. J Pineal Res 53: 129-137, 2012.

20. Zhang D, Yan H, Li H, Hao S, Zhuang Z, Liu M, Sun Q, Yang Y, Zhou M, Li K and Hang C: TGF $\beta$-activated kinase 1 (TAK1) inhibition by 5Z-7-oxozeaenol attenuates early brain injury after experimental subarachnoid hemorrhage. J Biol Chem 290: 19900-19909, 2015.

21. Ayer R and Zhang J: Connecting the early brain injury of aneurysmal subarachnoid hemorrhage to clinical practice. Turk Neurosurg 20: 159-166, 2010.

22. Kusaka G, Ishikawa M, Nanda A, Granger DN and Zhang JH: Signaling pathways for early brain injury after subarachnoid hemorrhage. J Cereb Blood Flow Metab 24: 916-925, 2004.

23. You WC, Wang CX, Pan YX, Zhang X, Zhou XM, Zhang XS, Shi JX and Zhou ML: Activation of nuclear factor- $\kappa B$ in the brain after experimental subarachnoid hemorrhage and its potential role in delayed brain injury. PLoS One 8: e60290, 2013.

24. Wu GS: The fuctional interactions between the p53 and MAPK signaling pathways. Cancer Biol Ther 3: 2156-2161, 2004. 
25. Liu R, Wu CX, Zhou D, Yang F, Tian S, Zhang L, Zhang TT and Du GH: Pinocembrin protects against $\beta$-amyloid-induced toxicity in neurons through inhibiting receptor for advanced glycation end products (RAGE)-independent signaling pathways and regulating mitochondrion-mediated apoptosis. BMC Med 10: 105, 2012.

26. Cahill J, Calvert JW, Marcantonio S and Zhang JH: p53 may play an orchestrating role in apoptotic cell death after experimental subarachnoid hemorrhage. Neurosurgery 60: 531-545, 2007.

27. Yang G, Zhang W, Qin Q, Wang J, Zheng H, Xiong W and Yuan J: Mono (2-ethylhexyl) phthalate induces apoptosis in p53-silenced L02 cells via activation of both mitochondrial and death receptor pathways. Environ Toxicol 30: 1178-1191, 2015.

28. Akaboshi M, Kawai K, Ujeno Y, Takada S and Miyahara T: Binding characteristics of (-)-(R)-2-aminomethylpyrrolidine (1,1-cyclobutanedicarboxylato)-2-platinum (II) to DNA, RNA and protein molecules in HeLa cells and its lethal effect: Comparison with cis- and trans-diamminedichloroplatinums(II). Jpn J Cancer Res 85: 106-111, 1994.
29. Tafolla E, Wang S, Wong B, Leong J and Kapila YL: JNK1 and JNK2 oppositely regulate p53 in signaling linked to apoptosis triggered by an altered fibronectin matrix: JNK links FAK and p53. J Biol Chem 280: 19992-19999, 2005.

30. Ljungman M: Dial 9-1-1 for p53: Mechanisms of p53 activation by cellular stress. Neoplasia 2: 208-225, 2000.

31. Ying GY, Jing CH, Li JR, Wu C, Yan F, Chen JY, Wang L, Dixon BJ and Chen G: Neuroprotective effects of valproic acid on blood-brain barrier disruption and apoptosis-related early brain injury in rats subjected to subarachnoid hemorrhage are modulated by heat shock protein 70/matrix metalloproteinases and heat shock protein 70/AKT pathways. Neurosurgery 79: 286-295, 2016.

32. Yin J, Li R, Liu W, Chen Y, Zhang X, Li X, He X and Duan C: Neuroprotective effect of protein phosphatase 2A/tristetraprolin following subarachnoid hemorrhage in rats. Front Neurosci 12: 96, 2018.

(i) $\Theta$ This work is licensed under a Creative Commons Attribution-NonCommercial-NoDerivatives 4.0 International (CC BY-NC-ND 4.0) License. 